\title{
A Prospective, Randomized Trial to Evaluate the Efficacy of Virtual Reality Treatment Versus Traditional Balance Treatment in Patients with Stroke
}

\author{
Zsanett Sipos $^{1,2}$, Zsófia Hőgye ${ }^{1}$ and Zsuzsanna Vekerdy-Nagy ${ }^{1,2}$ \\ 1. Department of Medical Rehabilitation and Physical Medicine, Clinical Center, University of Debrecen, Debrecen, 4032 Nagyerdei \\ Circuit 98, Hungary
}

2. Department of Pediatric Rehabilitation, the Bethesda Children Hospital of the Hungarian Reformed Church, Budapest, 1146

Bethesda Street 3, Hungary

\begin{abstract}
Recovery from stroke needs different time and speed in every case depending on the brain capacity of neural plasticity. Based on neurophysiological researches high repetition of practice is required to induce neuroplastic changes and functional recovery of motor deficits. However, data from earlier studies shows that stroke patients generally perform a very limited number of movement repetitions in traditional therapy sessions. Virtual Reality therapy was found to be beneficial in improving trunk, hand, upper and lower extremity functions. However, reviews found no definitive conclusions regarding balance. The present study focuses on evaluating the efficacy of Nintendo Wii ${ }^{\mathrm{TM}}$ on balance and gait functions of stroke patients. Twenty one hemiparetic stroke patients were randomly assigned to either control or therapy groups. Effectiveness was evaluated by Fugl-Meyer balance subtest, Functional Ambulation Categories (FAC), Romberg (R), sharpened Romberg (SR), one-legged stance (OLS), six-meter walking test (6MWT) and timed up and go test (TUG). The analysis revealed a significant effect of training with Nintendo Wii ${ }^{\mathrm{TM}}$ for FAC $\mathrm{z}=0.0267, \mathrm{FMBS} z=0.0046$, OLST $2 \mathrm{z}=0.0068$ and OLS1 $\mathrm{z}=0.0067$ (affected side). The control group demonstrated improvement in R $(\mathrm{z}=0.0474)$ and SR $(\mathrm{z}=$ 0.0411). In both groups improvement was detected in functional mobility and 6MWT (control group $z=0.0069$, Wii groupz $=0.0123$ ). No significant differences were found between groups. Findings of the current study support that balance treatment with Nintendo Wii ${ }^{\mathrm{TM}}$ is similarly effective method to improve static balance and functional mobility when compared with conventional therapy.
\end{abstract}

Key words: Nintendo Wii ${ }^{\mathrm{TM}}$, stroke, dynamic balance, rehabilitation, video game.

\section{Introduction}

Recovery of stroke patients is extremely heterogeneous and depends on the capacity of neural plasticity [1].

Balance between cell-intrinsic mechanisms and extrinsic regulatory molecules, that are controlled by activity-dependent processes of different kinds of interaction with the external world $[2,3]$ are behind the plastic properties of the brain [1]. Observational data showed that stroke patients generally perform a very limited number of movement repetition in traditional therapy sessions [4]. One proposed method for

Corresponding author: Zsanett Sipos, Ph.D. candidate, research fields: rehabilitation and neurorehabilitation. increasing the time of movement repetition is the virtual reality (VR) training. VR training is supposed to be a specific model of task-oriented motor learning. It is generally defined as technological intervention that alters properties of the physical world using multisensory stimulus, immediate sensory feedback on patients' movement and grading. Findings from animal studies suggested that optimalisation of motor learning [5-7] can be reached throughout immediate feedback and grading of tasks. Effectiveness of task specific training has been proven in the domains of activity. Walking speed and distance are increased [8]. In addition, research with animals and humans indicates that intensive task-oriented practice [1] induces cortical reorganisation $[9,10]$. Training in Enriched 
Environment (EE) results in better performance of functional tasks than training in basic environments [7, $11,12]$. Also $\mathrm{EE}$ has been found to strongly promote functional motor recovery $[1,6,7,12]$. Wii-based balance training has been found to be capable of stimulating cerebral plasticity $[1,14,15]$. Also changes in the reorganization of neural networks were observed in neurological diseases by VR [16]. VR therapy has been proven to be beneficial in body function and structure according to the International Classification of Functioning, disability and health model (ICF). It contributes to better hand [16], upper [17] and lower extremity functions [18]. Insufficient evidence was found about the effect on gait function and balance [6, 7]. A small number of randomized controlled trials (RCT) exist without clear evidence that virtual reality is more effective than "conventional therapy" (CT) in improving walking ability. As well as walking ability is one of the key element of the activity of daily living (ADL). This study focuses on evaluating the efficacy of Nintendo Wii ${ }^{\mathrm{TM}}$ on balance and gait functions in stroke patients. The purpose of this RCT was to compare VR therapy to conventional balance treatment (CBT) among hemiparetic stroke patients. We hypothesized that in patients with mild to moderate spasticity, balance training with Nintendo Wii could be superior to conventional interventions resulting in better outcomes of balance and gait functions.

\section{Methods}

This study received approval from the Human Research Ethics Committee of the University of Debrecen under process number HRB/052/00514-2/ 2013.

\subsection{Study Design}

A randomized controlled clinical trial was carried out at the Department of Medical Rehabilitation and Physical Medicine, Faculty of Medicine, University of Debrecen. Participants were randomly allocated to a control and an experimental group by draw. The flow of this study is shown in Fig. 1. The participants were evaluated in two visits: the first one for screening within one week before the randomization, the second one week after the last therapy session.

\subsection{Participants}

21 hemiparetic stroke patients took part in the study, ten in the control and eleven in the experimental group.

\subsection{Inclusion Criteria}

Patients with residual hemiparesis caused by stroke with mild to moderate spasticity in lower extremity muscles (MAS 1-3), good muscle strength (at least 3 in a five point rating scale) in the antigravity muscles (gluteal muscles - medius and maximus - and femoral quadriceps) and independent walking ability indoor with or without assistive device were included.

\subsection{Intervention}

Patients met the inclusion criteria after the initial evaluation were randomly allocated to 2 groups. Both groups participated in 30 minutes balance therapy per day every weekday in a two-week period. The control group received conventional balance therapy focusing on trunk stabilization (Proprioceptive Neuromuscular Facilitation) and weight shifting in light of patients' ability in the same time frame. The experimental group was assigned to balance training with Nintendo Wii ${ }^{\mathrm{TM}}$ Fit gaming system to practise balance and improve postural gait stability. During the intervention, four games were carried out in order to train balance and coordination (Heading, TableTilt, Penguin Slide and Tight Rope) each was repeated three times under the supervision of a physiotherapist. Besides this, both groups were treated with conventional physical therapy (muscle strengthening, endurance exercise and practicing functional movements) for one hour every weekday.

\subsection{Measurements}

Effectiveness was evaluated by functional mobility, 


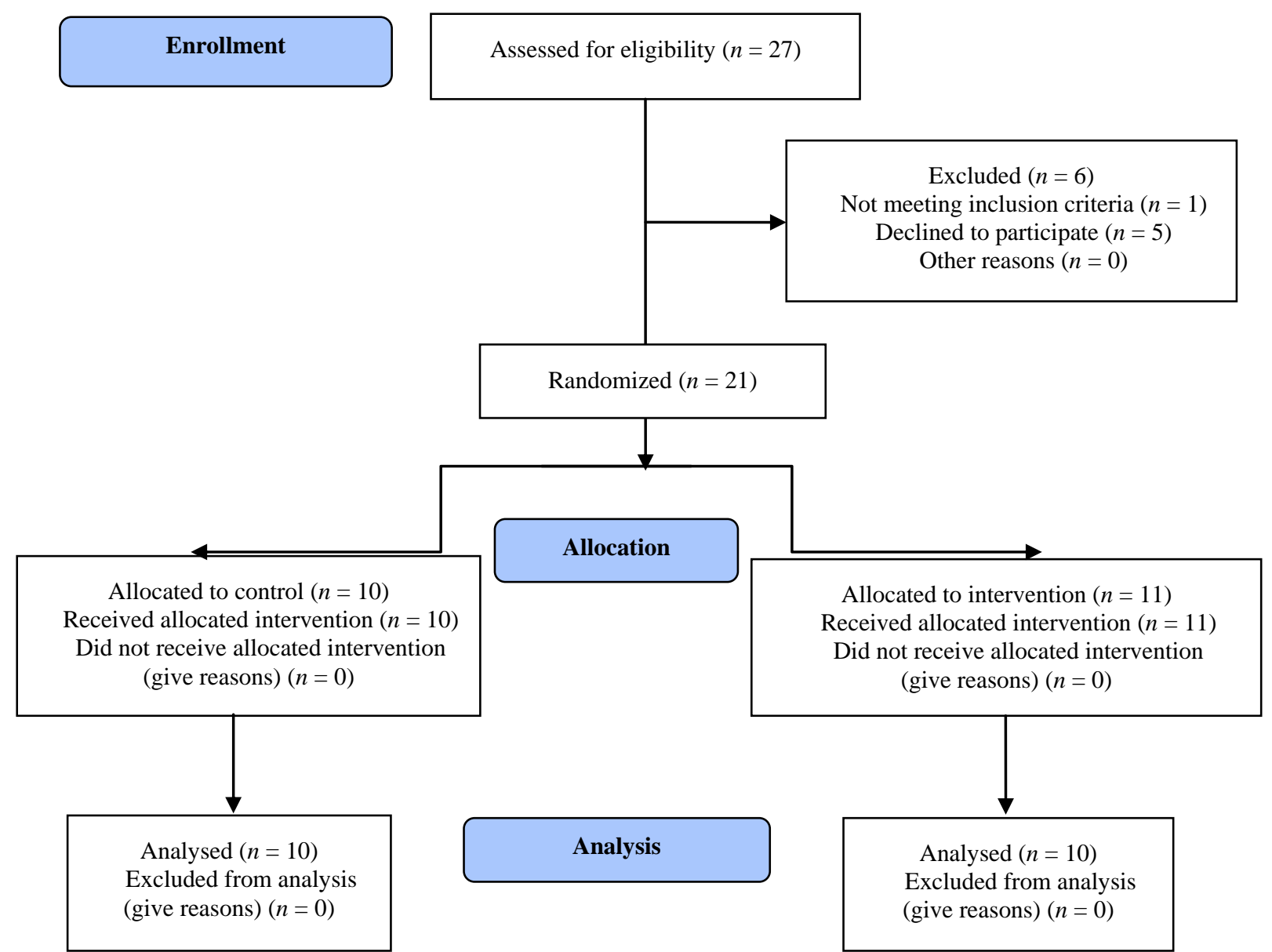

Fig. 1 Four phase flow-diagram for study design, showing the number of patients enrolled, screened, randomized and analysed.

dynamic and static balance test. All participants completed the Fugl-Meyer balance subtest (FMBS), Functional Ambulation Categories (FAC), one-legged stance (OLS), Romberg (R), sharpened Romberg (SR), six-meter walking test (6MWT) and timed up and go test (TUG). The primary outcomes were the Fugl-Meyer balance subtest, Functional Ambulation Categorization (FAC) and measurement of functional mobility (6MWT, TUG). The secondary outcomes were the static balance tests (R, SR, OLS).

\subsection{Statistical Analysis}

Data analysis was done by the Statistical Package in the Social Science (SPSS system) version 18. Scores of clinical scales, age and sex were summarized using non-parametric statistics, reporting mean, standard deviation (SD), min and max. The data were compared between the two groups using Mann-Whitney U test at baseline for verifying the homogeneity of the two groups and at the end of treatments for assessing the potential differences in changes. Also data were analyzed for differences in the changes within groups by Wilcoxon signed rank test. In all analyses, the nullhypothesis was rejected when the probability value was less than (or equal to) 0.05 .

\section{Results}

\subsection{Epidemiological and Clinical Characteristics of the Patients}

21 stroke patients participated in the study 14 men ( $N=7$ in the Wii group) and 7 ( $N=4$ in the Wii group) women. The mean age was 59.45 years (range 37 to 78 years). All participants had spastic hemiparesis, twelve 
of them on the right-side (7 and 5 in Wii and control groups, resp.). There were no significant imbalances between groups with respect to gender and affected side. Patients randomized to the control and Wii groups were almost at the same age (mean age 58.11 years versus 60.55 years) and did not have significant differences in the static ( $R, S R$,$) and dynamic balance$ function (FMBS and TUG) at baseline. There were small differences between the control and the Wii groups in the OLST1, OLST2 and walking distance (6MWT). The control group achieved 4 and the Wii group presented 3 in FAC at baseline. However, the differences between the two groups were not significant in these parameters. Baseline characteristics are presented in Table 1.

\subsection{Primary and Secondary Outcomes}

The analysis revealed a significant effect of training with Nintendo Wii ${ }^{\text {TM }}$ for FMBS z $=0.0046$ and FAC z $=0.0267$. Moreover, meaningful improvement was found in secondary outcomes, OLST2 $\mathrm{z}=0.0068$ and OLS1 $\mathrm{z}=0.0067$ (affected side) as well. The control group demonstrated improvement in static balance, $\mathrm{R}$ $(z=0.0474)$ and SR $(z=0.0411)$. In both groups, we observed an improvement in functional mobility, 6MWT (control group $\mathrm{z}=0.0069$, Wii group $\mathrm{z}=$ 0.0123 ) (Table 2).

We didn't find significant differences between groups. Remarkable improvement was detected in OLST1 (139.5 versus $91.5 \mathrm{z}=0.1840)$, OLST2 (121.5 versus $109.5 \mathrm{z}=0.9714$ ) in the therapy group with larger non-significant changes in OLST1. Significant improvement was found in FAC (127.5 versus $103.5 \mathrm{z}$ $=0.5973)$, but the changes were not significantly higher and FMBS (141.4 versus $89.5 \mathrm{z}=0.1352$ ) with greater non-significant changes in the therapy group. We found meaningful improvement in $\mathrm{R}$ (114 versus $116 \mathrm{z}$ $=0.5854)$ and $\operatorname{SR}(109$ versus $122 \mathrm{z}=0.3949)$ in the control group. However, the changes were not superior above the training group. Significant improvement was

Table 1 Baseline epidemiological data of 21 spastic hemipateric stroke patients.

\begin{tabular}{llll}
\hline & $\begin{array}{l}\text { Control group } \\
n=10\end{array}$ & Wii group \\
$n=11$ & $\begin{array}{l}\text { Significance } \\
\text { Mann-Whitney U test (z) }\end{array}$ \\
\hline FMBS & $10 \pm 2.54$ & $8.36 \pm 2.20$ & 0.1105 \\
FAC & $3.9 \pm 0.88$ & $3.36 \pm 0.67$ & 0.1690 \\
OLST1 (affected side) & $6.1 \pm 9.904$ & $2.45 \pm 2.84$ & 0.8855 \\
OLST2 (non-affected side) & $9 \pm 11.33$ & $3.91 \pm 4.72$ & 0.1886 \\
R (sec) & $19.5 \pm 13.95$ & $19.27 \pm 14.89$ & 0.9352 \\
SR (sec) & $6.05 \pm 9.53$ & $4.28 \pm 5.38$ & 0.9713 \\
6MWT & $162.55 \pm 126.4$ & $150.7 \pm 83.56$ & 0.6983 \\
TUG (sec) & $19.74 \pm 9.92$ & $17.91 \pm 10.59$ & 0.3071 \\
\hline
\end{tabular}

Note: FMBS = Fugl-Meyer balance subtest, FAC = Functional Ambulation Categories, Romberg (R), sharpened Romberg (SR), OLST $=$ one-legged stance test, $6 \mathrm{MWT}=$ six-meter walking, $\mathrm{TUG}=$ timed up and go test.

Table 2 Summary training effect for therapy and control group—primary and secondary outcomes.

\begin{tabular}{lllllll}
\hline & \multicolumn{3}{c}{ Wii group } & \multicolumn{3}{c}{ Control group } \\
\cline { 2 - 7 } & 1st measurement & 2nd measurement & $P$ & 1st measurement & 2nd measurement & $P$ \\
\hline FMBS & $8.36 \pm 2.20$ & $9.82 \pm 2.04$ & 0.0046 & $10 \pm 2.54$ & $10.8 \pm 1.93$ & 0.0872 \\
FAC & $3.36 \pm 0.67$ & $4 \pm 1.09$ & 0.0267 & $3.9 \pm 0.88$ & $4.5 \pm 1.17$ & 0.0842 \\
OLST1 & $2.45 \pm 2.84$ & $6.18 \pm 8.097$ & 0.0067 & $6.1 \pm 9.904$ & $7.7 \pm 11.96$ & 0.0872 \\
OLST2 & $3.91 \pm 4.72$ & $8.64 \pm 10.698$ & 0.0068 & $9 \pm 11.33$ & $17.72 \pm 12.73$ & 0.0632 \\
R & $19.27 \pm 14.89$ & $24.9 \pm 10.26$ & 0.0843 & $19.5 \pm 13.95$ & $27.2 \pm 9.17$ & 0.0474 \\
SR & $4.28 \pm 5.38$ & $9.73 \pm 9.52$ & 0.1302 & $6.05 \pm 9.53$ & $13.65 \pm 13.47$ & 0.0411 \\
6MWT & $150.7 \pm 83.56$ & $194.7 \pm 93.35$ & 0.0123 & $162.55 \pm 126.4$ & $202.7 \pm 133.45$ & 0.0069
\end{tabular}

Note: FMBS = Fugl-Meyer balance subtest, FAC = Functional Ambulation Categories, Romberg (R), sharpened Romberg (SR), OLST $=$ one-legged stance test, $6 \mathrm{MWT}=$ six-meter walking. 
found in 6MWT (125.5 versus $105.5 \mathrm{z}=0.7509)$ in both groups. Wii Fit training and conventional balance therapy was similar in improving walking distance.

\section{Discussion}

In general, both groups showed improvement in balance. The intra-group analysis revealed significant improvement in all of the primary outcome scales' score (FMBS, FAC, 6MWT), OLST1 and OLST2 in the therapy group. Compare to conventional balance therapy, Wii Fit gaming is effective on walking ability (FAC) and functional balance (FMBS) improvements in our study group. In an earlier study, partial benefits were observed in walking ability recovery on the follow-up [19]. There is low and very low evidence that VR therapy improves walking ability $[6,7,13,19]$. According to our findings, training with Nintendo Wii ${ }^{\mathrm{TM}}$ on postural balance probably had positive effects on reducing the need for aids and/or supervision during walking for avoiding the risk of falling [19].

Both groups showed significant improvement in functional mobility (6MWT). Regarding this, we didn't detect statistically significant differences between the groups, maybe because auditory and visual feedback similarly improves overall independence [20]. An earlier review showed insufficient evidence to draw conclusions on whether a virtual reality approach was more effective in improving gait speed than conventional therapy $[6,7]$. Also a meta analysis for physical therapy poststroke didn't find significant effect on comfortable gait speed, maximum gait speed and walking ability $[13,14]$. Findings of the current study support that a video game-based therapy could be effective in enhancing balance and walking speed (6MWT) similarly to a conventional balance therapy.

Similarly to our findings, Barcala and co-workers found significant improvement in functional mobility, static and functional balance in their RCT, but no statistically significant differences between the experimental and control group [21]. Contrary to our findings, an RCT showed that improvements in functional balance were significantly higher in Wii group [22]. Also Wii Fit training proved to be more effective than usual balance therapy in improving functional balance (Berg Balance Schale) and independence in activity of daily living (Barthel index) [19]. A review presented by Lohse and coworkers demonstrated significant moderate advantage in activity $[13,14,19]$.

\section{Conclusion}

The purpose of this study was to investigate the efficacy of balance training on functional balance using video game-based intervention. Although it is difficult to draw clinical implication based on the results of small sample size, it is possible the balance treatment with Nintendo Wii is similarly effective method to improve static balance and functional mobility comparing to conventional therapy.

In conclusion, a Wii-based approach is promising for post-stroke balance rehabilitation. The therapy performed with a Nintendo Wii could be an alternative way to treat balance among stroke patients because of its several advantages: cost effectiveness, usage in out-patient rehabilitation and more fun to do (which is important for the patient's engagement in the therapy). Motivation of the patients is important throughout the rehabilitation process and repetition is also crucial (for plasticity). Several questions remain unclear about intensity, timing, and mode of action. A larger randomized controlled trial is recommended to further investigate.

\section{Acknowledgements}

The authors wish to thank Attilla Nagy MD, who is assistant lecturer in the Division of Biostatistics and Epidemiology, Department of Preventive Medicine, Faculty of Public Health, Medical and Health Science Centre, University of Debrecen, Hungary, for the statistical analysis. Also we would like to thank the staff of the Department of Medical Rehabilitation and Physical Medicine. 


\section{References}

[1] Faralli, A., Bigoni, M., Mauro, A., Rossi, F., and Carulli, D. 2013. "Noninvasive Strategies to Promote Functional Recovery after Stroke.” Neural Plasticity 2013: 854597.

[2] Foscarin, S., Rossi, F., and Carulli, D. 2012. "Influence of the Environment on Adult CNS Plasticity and Repair." Cell and Tissue Research 349 (1): 161-7.

[3] Rossi, F., Gianola, S., and Corvetti, L. 2007. "Regulation of Intrinsic Neuronal Properties for Axon Growth and Regeneration.” Progress in Neurobiology 81 (1): 1-28.

[4] Lang, C. E., Macdonald, J. R., Reisman, D. S., Boyd, L., Jacobson Kimberley, T., Schindler-Ivens, S. M., Hornby, T. G., Ross, S. A., and Scheets, P. L. 2009. “Observation of Amounts of Movement Practice Provided During Stroke Rehabilitation." Archives of Physical Medicine and Rehabilitation 90 (10): 1692-8.

[5] Sveistrup, H. 2004. "Motor Rehabilitation Using Virtual Reality." Journal of Neuroengineering and Rehabilitation 1 (1): 10.

[6] Laver, K. E., George, S., Thomas, S., Deutsch, J. E., and Crotty, M. 2011. "Virtual Reality for Stroke Rehabilitation." The Cochrane Database of Systematic Reviews 9 (9): CD008349.

[7] Laver, K. E., George, S., Thomas, S., Deutsch, J. E., and Crotty, M. 2015. "Virtual Reality for Stroke Rehabilitation." The Cochrane Database of Systematic Reviews 2 (Feb.): CD008349.

[8] French, B., Thomas, L. H., Leathley, M. J., Sutton, C. J., McAdam, J., Forster, A., Langhorne, P., Price, C. I., Walker, A., and Watkins, C. L. 2007. "Repetitive Task Training for Improving Functional Ability after Stroke." The Cochrane Database of Systematic Reviews 4 (4): CD006073.

[9] Nudo, R. J., Milliken, G. W., Jenkins, W. M., and Merzenich, M. M. 1996. "Use-Dependent Alterations of Movement Representations in Primary Motor Cortex of Adult Squirrel Monkeys." The Journal of Neuroscience: The Official Journal of the Society for Neuroscience 16 (2): 785-807.

[10] Nudo, R. J., Plautz, E. J., and Frost, S. B. 2001. "Role of Adaptive Plasticity in Recovery of Function after Damage to Motor Cortex." Muscle \& Nerve 24 (8): 1000-19.

[11] Risedal, A., Mattsson, B., Dahlqvist, P., Nordborg, C., Olsson, T., and Johansson, B. B. 2002. "Environmental Influences on Functional Outcome after a Cortical Infarct in the Rat." Brain Research Bulletin 58 (3): 315-21.

[12] Zhao, L. R., Risedal, A., Wojcik, A., Hejzlar, J., Johansson, B. B., and Kokaia, Z. 2001. "Enriched Environment Influences Brain-derived Neurotrophic
Factor Levels in Rat Forebrain after Focal Stroke." Neuroscience Letters 305 (3): 169-72.

[13] Lohse, K. R., Hilderman, C. G., Cheung, K. L., Tatla, S., and Van der Loos, H. F. 2014. "Virtual Reality Therapy for Adults Post-stroke: A Systematic Review and Meta-analysis Exploring Virtual Environments and Commercial Games in Therapy." PLoS One 9 (3): e93318.

[14] Veerbeek, J. M., van Wegen, E., van Peppen, R., van der Wees, P. J., Hendriks, E., Rietberg, M., and Kwakkel, G. 2014. "What is the Evidence for Physical Therapy Poststroke? A Systematic Review and Meta-analysis." PLoS One 9 (2): e87987.

[15] Omiyale, O., Crowell, C. R., and Madhavan, S. 2015. "Effect of Wii-based Balance Training on Corticomotor Excitability Post Stroke." Journal of Motor Behavior 47 (3): 190-200.

[16] Gatica-Rojas, V., and Mendez-Rebolledo, G. 2014. "Virtual Reality Interface Devices in the Reorganization of Neural Networks in the Brain of Patients with Neurological Diseases." Neural Regeneration Research 9 (8): 888-96.

[17] Henderson, A., Korner-Bitensky, N., and Levin, M. 2007. "Virtual Reality in Stroke Rehabilitation: A Systematic Review of Its Effectiveness for Upper Limb Motor Recovery." Topics in Stroke Rehabilitation 14 (2): 52-61.

[18] Deutsch, J. E., Brettler, A., Smith, C., Welsh, J., John, R., Guarrera-Bowlby, P., and Kafri, M. 2011. "Nintendo Wii Sports and Wii Fit Game Analysis, Validation, and Application to Stroke Rehabilitation." Topics in Stroke Rehabilitation 18 (6): 701-19.

[19] Morone, G., Tramontano, M., Iosa, M., Shofany, J., Iemma, A., Musicco, M., Paolucci, S., and Caltagirone, C. 2014. "The Efficacy of Balance Training with Video Game-based Therapy in Subacute Stroke Patients: A Randomized Controlled Trial." BioMed Research International 2014: 580861.

[20] Barclay-Goddard, R., Stevenson, T., Poluha, W., Moffatt, M. E., and Taback, S. P. 2004. "Force Platform Feedback for Standing Balance Training After Stroke." The Cochrane Database of Systematic Reviews 4 (4): CD004129.

[21] Barcala, L., Grecco, L. A., Colella, F., Lucareli, P. R., Salgado, A. S., and Oliveira, C. S. 2013. "Visual Biofeedback Balance Training Using Wii Fit after Stroke: A Randomized Controlled Trial." Journal of Physical Therapy Science 25 (8): 1027-32.

[22] Bower, K. J., Clark, R. A., McGinley, J. L., Martin, C. L., and Miller, K. J. 2014. "Clinical Feasibility of the Nintendo Wii for Balance Training Post-stroke: A Phase II Randomized Controlled Trial in an Inpatient Setting." Clinical Rehabilitation 28 (9): 912-23. 